\title{
Presentation of multiple midface fractures combined with intracranial injury treated conservatively
}

\author{
Talal Rane ${ }^{1}$, Firas Nasser ${ }^{2}$, Samir Taha $^{2^{*}}$ \\ ${ }^{1}$ Department of Oral \& Maxillofacial Surgery, Al-Ahli Hospital, Doha, Qatar \\ ${ }^{2}$ Department of Oral and Maxillofacial Surgery, Hamad Medical Corporation, Doha, Qatar; *Corresponding Author: staha3@hmc.org.qa
}

Received 17 July 2013; revised 19 August 2013; accepted 1 September 2013

Copyright (C 2013 Talal Rane et al. This is an open access article distributed under the Creative Commons Attribution License, which permits unrestricted use, distribution, and reproduction in any medium, provided the original work is properly cited.

\begin{abstract}
Midface injuries are normally the result of high forces impacted on the face, that can bypass the power of the connection buttresses, which are thick and strong, base of skull is also a rigid and tough structure which requires significant forces to break. In our case, multiple midface and cranial base fractures are presented, which reflect injury caused by high forces and normally, these result in instability in the facial skeleton, or cause CSF rhinorrhea, or sharp spicules to endanger the orbit or the anterior cranial fossa, or the presence of any other indication of surgery. It is unusual to find such an extent of facial trauma and such a multiplication of fractures on the midface and cranial base levels, yet they were stable enough and without a real indication to require surgical interference.
\end{abstract}

Keywords: Midface Fracture; Upper Face Fracture; Conservative Management

\section{INTRODUCTION}

Combined intracranial injury with midface and upper face multiple fractures signify severe force involved [14], published work on forces calculated to produce a fracture of each craniofacial bone structure is well documented $[1,2]$, normally, this results in severe injuries to the face [1-3]. Hodgson, V.R. et al. presented detailed work on the tolerance of facial bones to impact [3], and indicated that impacts produce fracture near 200 pounds, but that is closely related to the length of the pulse of impact measured in milliseconds.

A lot of work is presented in the literature about treatment [5-8], sequencing of which bone to fix first [6,7], timing of treatment $[6,7]$.

We present a case of facial trauma that resulted in multiple midface and upper face fractures combined with a base-of-skull fracture, but all were undisplaced and not mobile therefore, did not require any intervention, and were managed conservatively, together with the intracranial hemorrhage being minor, that did not require surgical treatment as well.

\section{CASE REPORT}

A (46) years old year old male was brought to the A \& E Department of Hamad General Hospital on 12 May 2009, he was lowering a swimming pool basin down into a pre-dug site in a house garden, when the edge of the basin hit him on the face.

Patient was conscious, oriented with GCS 15/15, his BP was 146/82 and Oxygen saturation of 98\%, airway was stable and neck is clear of injury.

Craniofacial examination revealed bilateral periorbital ecchymosis, active oronasal bleeding, and oro-facial lacerations.

Pupils were round, equal and brisk reactive, directly and consensually, visual acuity intact with mild proptosis of right eye, no obvious restriction of ocular movements.

CT scan showed left small foci of frontal subarachnoid hemorrhage and bleeding into the anterior part of the interhaemospheric fissure and very small pneumocephalus of the frontal region (Figure 1) plus fracture of the anterior cranial fossa, both orbital roofs (Figures 1 and 2) and right side of the frontal bone (Figure 3) with fracture of the left zygoma and a lefort I (Figure 2), nasal bone, both medial walls and floors of orbits (Figures 2 and 3), as reported on CT by radiologist and confirmed by the maxillofacial team responsible for patient.

Initially patient was managed in the trauma room by bilateral anterior nasal packs, and oro-facial lacerations were closed as appropriate, under an umbrella of intravenous Augmentin plus Dexamethasone, patient was shifted to Trauma ITU for neurological and eye observation. 


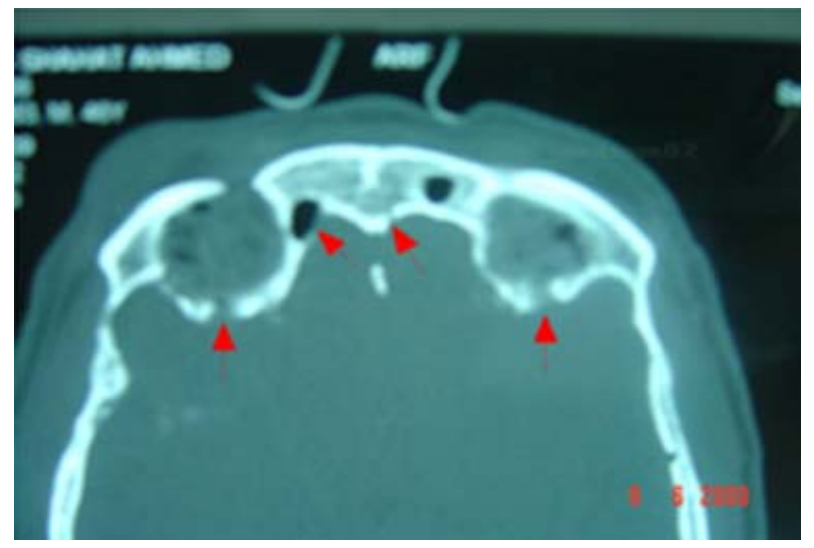

Figure 1. Fractures seen in orbits and posterior wall of frontal sinus, small pneumocephalus.

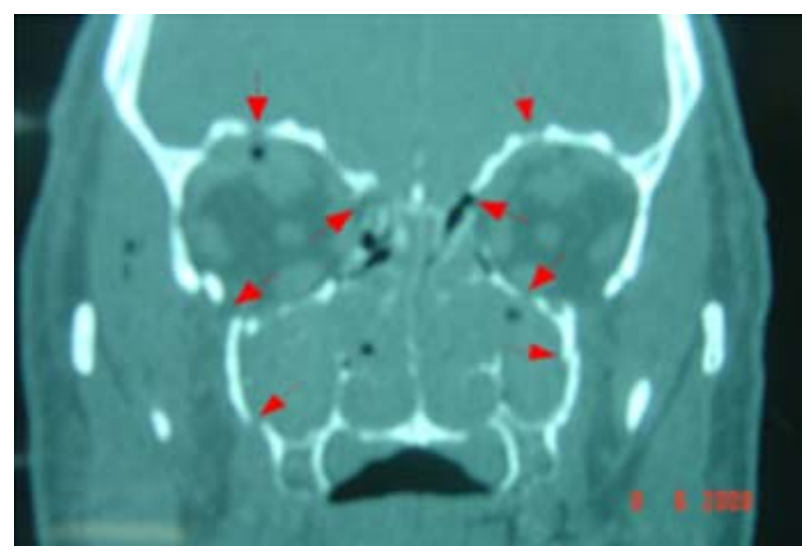

Figure 2. Fractures in both medial walls, both floors, both orbital roofs, lefort I level.

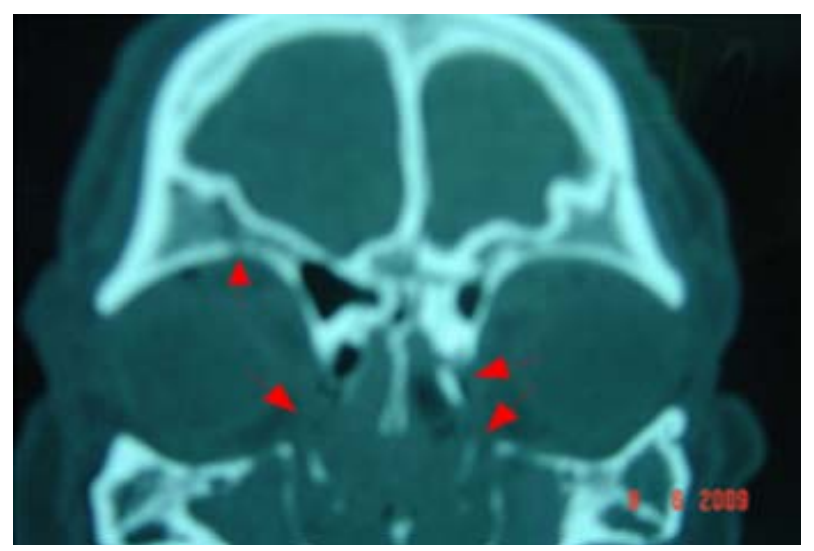

Figure 3. Fractures seen in orbital floor, roof, medial wall.

Secondary re-assessment revealed that interestingly all facial fractures were undisplaced and mobility of the midface was very minimal with no apparent impact on vision, aesthetics, nor occlusion, and therefore, did not warrant any maxillofacial surgical intervention, and patient was treated conservatively with antibiotics, Dexamethasone for three days, and close monitoring.
Full recovery was achieved without any functional, aesthetic, or neurological deficit. Recovery time was around 3 weeks from injury, which is time necessary for bone healing to achieve stability, surgical intervention on the other hand would have been attempted after at least 48 hours of neurological observation to ensure that he is neurologically fit to undergo a non-life saving major surgery, also, midface repair is generally done after few days to allow edema to subside first [6,7], taking the above into consideration, one week of waiting is expected plus one week of post op recovery is needed, and this phase might be slightly quicker than the period necessary for conservative treatment, but the time difference is small enough not to pull the decision making one way or the other.

\section{DISCUSSION}

The patient was placed on soft diet for the period necessary for bone initial phase which was 3 weeks, surgical intervention affords faster rehabilitation to normal diet, but on the other hand, predisposes to all the surgical morbidities associated with major procedure, which should be avoided if surgical treatment is not the only option, generally soft diet restriction in case of open reduction is only applied for few days.

Other post op issues would be neurological monitoring for 48 - 72 hours initially, and long term out-patient based follows up to monitor any frontal sinus pathology, intracranial pathology, exclude signs of developing meningeal disease. These issues have to be monitored equally whether surgical or conservative treatment followed.

Literature review shows that not all midface fractures need surgery $[9,10]$. Conservative treatment however seems to be a much less likely modality in the case of midface trauma, Adeyemo, W.L. and co-workers estimated that to be around $2.6 \%$ of all cases [11]. It is also known that fractures of the posterior wall of the frontal sinus need cranialization, unless undisplaced and not associated with CSF rhinorrhea or nasofrontal duct injury [12], this case presented as a wide magnitude of injury which went to involve most facial bones and skull base, i.e. midface and upper face fractures. This is normally caused by high force or known as high velocity injuries $[1,3]$, yet none of the bones of the face required surgery as they were non displaced nor mobile, which made conservative management possible and indeed, indicated, therefore careful assessment and reassessment after most of the initial edema has subsided, has a lot to command for in the management of facial injuries.

\section{REFERENCES}

[1] Hudson, et al. (1997) Experimentally induced upper fa- 
cial third fractures in unembalmed human cadaver heads. The Journal of Trauma, Injury, Infection, and Critical Care Issue, 42, 705-710. http://dx.doi.org/10.1097/00005373-199704000-00021

[2] Nahum, A. and Melvin, J. (2001) Accidental injury, biomechanics and prevention. 276, Table 12.3.

[3] Hodgson, V.R. (1967) Tolerance of the facial bones to impact. American Journal of Anatomy, 120, 113. http://dx.doi.org/10.1002/aja.1001200109

[4] Crandall, J.R., Kuhlmann, T.P. and Pilkey, W.D. (1995) Air and knee bolster restraint system: laboratory sled tests with human cadavers and the hybrid III dummy. Journal of Trauma, 38, 517-520. http://dx.doi.org/10.1097/00005373-199504000-00009

[5] Louis, P.J. (2004) Oral and maxillofacial surgery (Peterson). Management of Pan Facial Fractures, 8.

[6] Moe K.S. (2012) Facial trauma, management of panfacial fractures.
[7] (2009) Head and neck surgery. University of Washington School of Medicine.

[8] Wenig, B.L. (1991) Management of panfacial fractures. Otolaryngologic Clinics of North America, 24, 93-101.

[9] Tian, W., Li, S., Pan, J., Gao, Z. and Zheng, X. (1999) Surgical reduction and rigid internal Fixation of midface fractures. 17, 136-139.

[10] Johnson, W. (2001) Oral and maxillofacial surgery. Fonseca, 4, Chapter 8.

[11] Adeyemo, W.L., Taiwo, O.A., Ladeinde, A.L., Ogunlewe, M.O., Adeyemi, M.O. and Adepoju, A.A. (2012) Mid-facialfractures: A 5-year retrospective review in a Nigerian teaching hospital. Nigerian Journal of Medicine, 21, 3135.

[12] Luce, E.A. (1987) Frontal sinus fractures, guidelines to management. Plastic and Reconstructive Surgery, 80, 500. http://dx.doi.org/10.1097/00006534-198710000-00003 